\title{
The Role of Telenursing in Improving the Chronic Diseases Management
}

\begin{abstract}
Introduction: Due to the growing trend of aging and chronic diseases in the country in order to reduce the complications of aging and disease, tele-nursing is the use of telecommunications technology to provide care and counseling to patients. Therefore, the aim of this study was to investigate the role of telenursing in the management of chronic diseases in the elderly.

Methods: The present study was performed in a systematicreview and by searching for articles using the keywords Tele Nursing elderly, Chronic Diseases and their combination in SID, Magiran and Science Direct, PubMed and Scopus Persian databases. Title and abstract of articles and elimination of duplicates, quality of articles found using JBI tool by researchers, reviewed and finally 43 articles were included in this study.

Results: The effective role of tele-nursing in improving disease management in patients with type 2 diabetes, hypertension, nonalcoholic fatty liver, heart failure was seen in the reviewed articles and a study that violated the results was not found in the reviewed articles.

Conclusion: Telenursing plays an effective role in the management of chronic diseases, so the use of this technology in the country and providing a bed for the proper use of the elderly is important because it can result. Have desirable benefits, such as reducing the costs of the health care system and long-term hospitalization, in which case it is necessary to strengthen the infrastructure and allocate sufficient funds.
\end{abstract}

Keywords: Remote Nursing, Chronic Diseases, Elderly

\footnotetext{
Affiliations

1- MSC Nursing, Instructor, Department of Internal Medicine and Surgery, School of Nursing, Aja University of Medical Sciences. Tehran, Iran.

Corresponding author: Haj sh 13@yahoo.com

2- PhD student in Nursing, Department of Special Care, Iran University of Medical Sciences, Tehran, Iran.

3- Researcher, Armed forces health administration of Islamic Republic of Iran, Tehran, Iran.
}

Article Info

Authors:

Ahmadi $\mathrm{Y}^{1 *}$ •

AlazmaniNodeh $\mathrm{F}^{2}$ 우

Maleki $\mathrm{H}^{3}$ 을

Received: 2020/06/04

Accepted: 2020/07/31

E-Published: 2020/09/18

\section{Citation Subjects:}

Telemedicine, Telenursing, Chronic Illness, Aged 
فصلنامه يرستار و پيزشك در رزم / تابستان 99 / سال هشتم

\section{نقش تلهنرسينَ در بهبود مديريت بيمارىهاى مزمن}

\section{اطلاعات مقاله

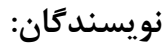

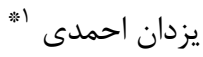

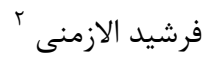 \\ همايون ملكى برني الازنى}

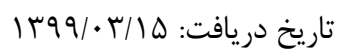

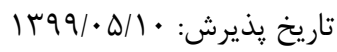

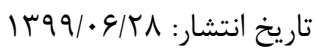

وازَّان كليدى: يرستارى از راه دور، بيمارى هاى مزمن، سالمند

مقدمه: با توجه به روند رو به رشد سالمندى و ابتلا به بيمارىهاى مزمن در كشور در

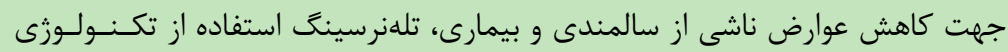

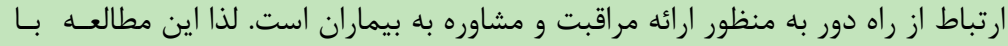
هدف بررسى نقش تلهنرسينگ در مديريت بيمارىهاى مزمن در سالمندان انجام شد.

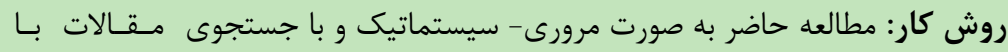

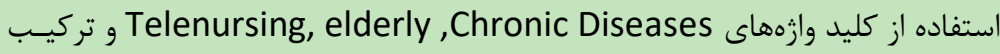

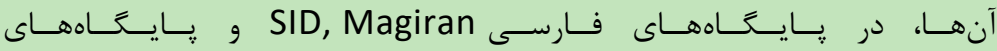

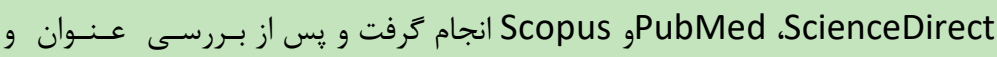

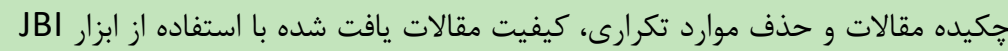

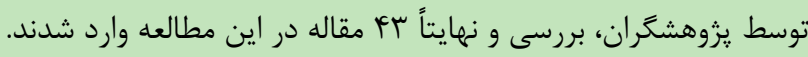

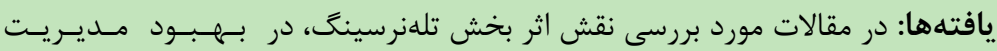

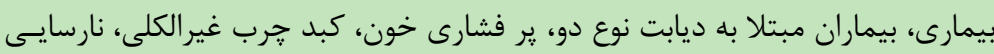

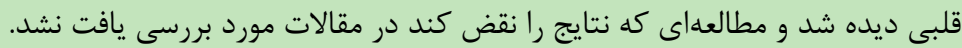

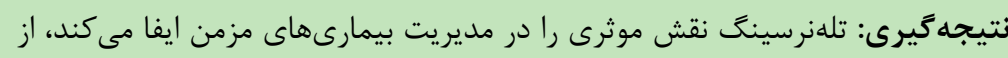

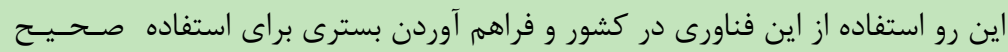

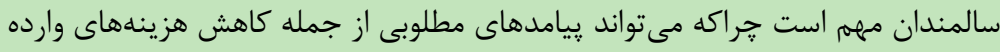

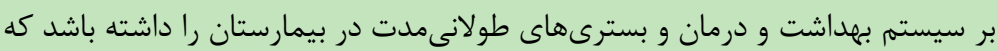
در اين خصوص نياز به تقويت زيرساختها و اختصاص بودجه كافى دارد.

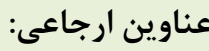

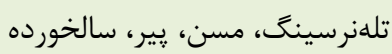

$$
\text { وابستكى سازمانى نويسندكان }
$$

1- كارشناس ارشد يرستارى، مربى هيئت علمى، كروه داخلى و جراحى، دانشكده يُرستارى، دانشَّاه علوم يزشكى ارتش جمهورى اسلامى ايران تهران ايران.

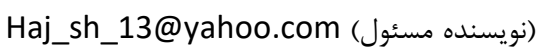

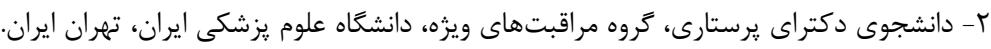

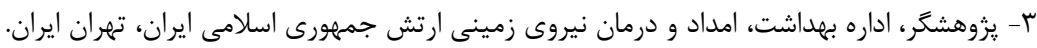


به افزايش كيفيت و بهبود دسترسى به مراقبتهاى بهداشتى و و

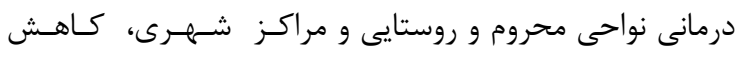

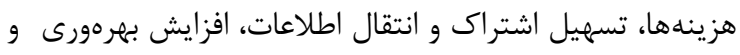

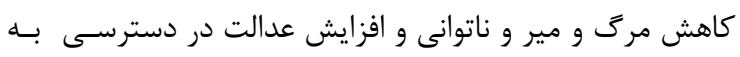

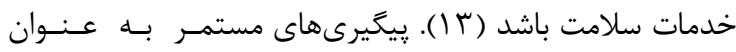
بخشى اساسى از سرويس هاى مراقبتى محسوب شده و احتمـال بـدي

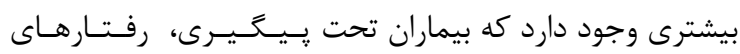

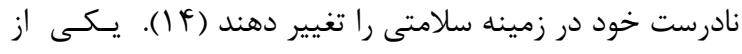

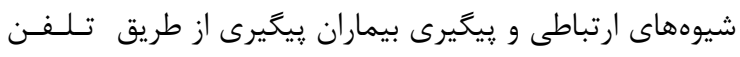

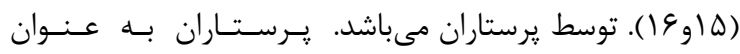

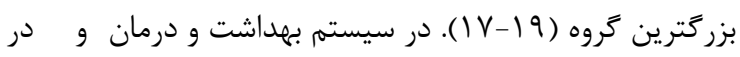

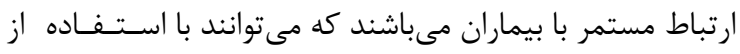

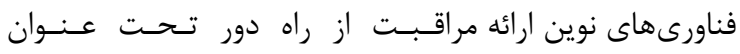

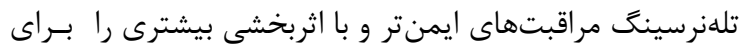

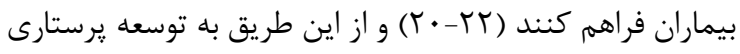

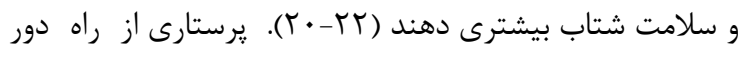

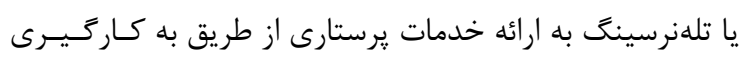

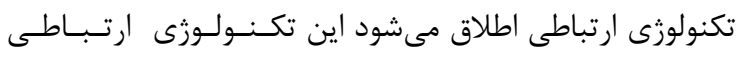

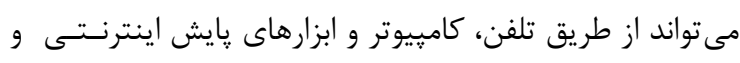

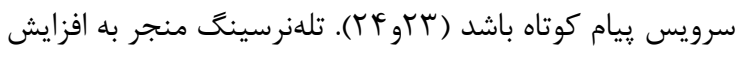

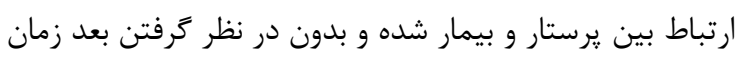

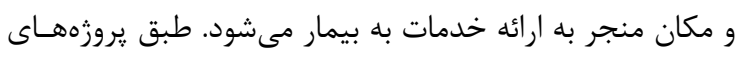

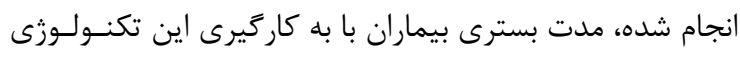

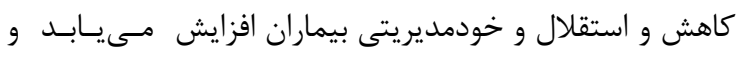

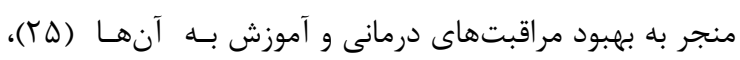

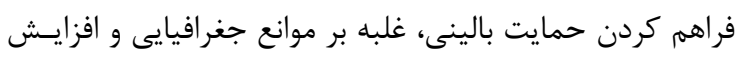

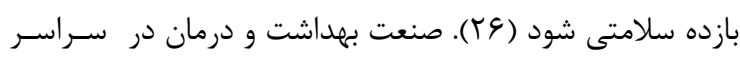

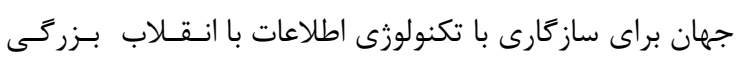

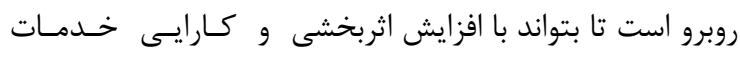

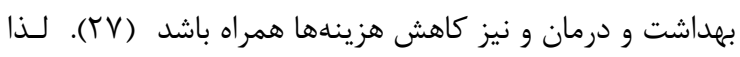

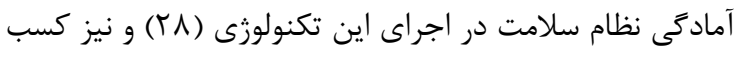

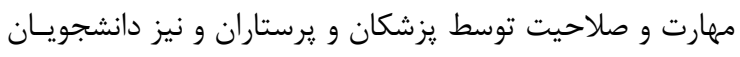

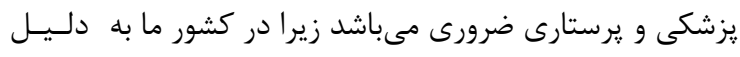

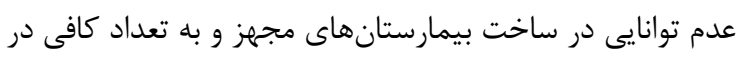

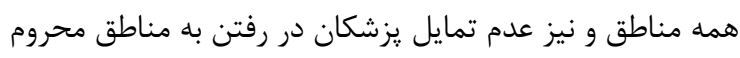

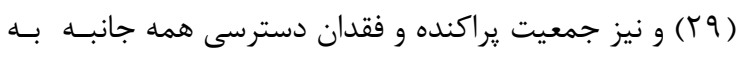

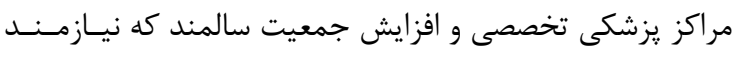

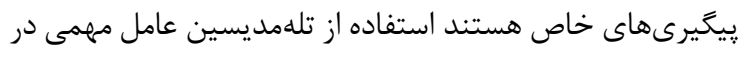

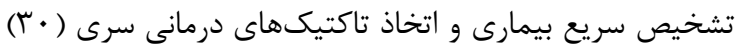

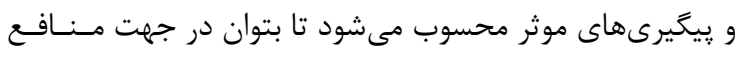

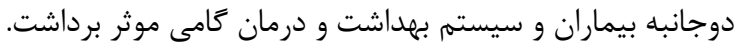

2- Telenursing
مقدمه

جهان امروز با استفاده از فناورى اطلاعات و تكنولوزىهاى نوين ارتباطى و ريشرفت سريع با تغييرات اساسى مواجه اسـت (1) (1).

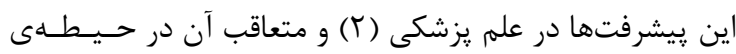

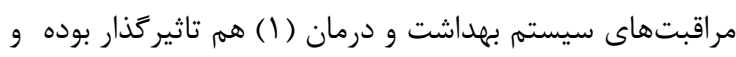

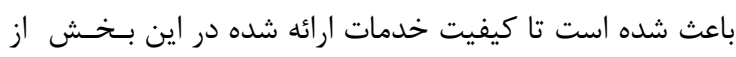

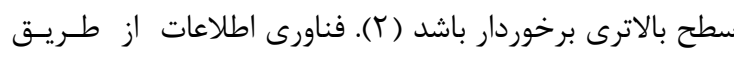
روشهاى مختلف مىتواند در حوزمى سلامت كارا بـاشـد (Y).

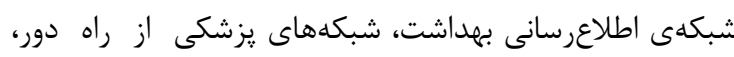

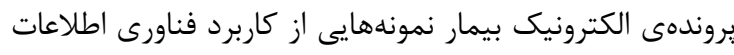

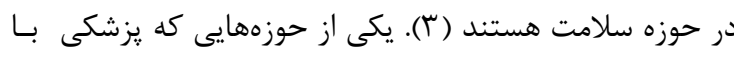

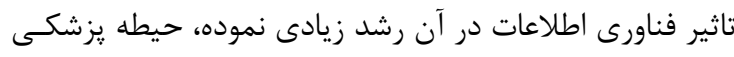

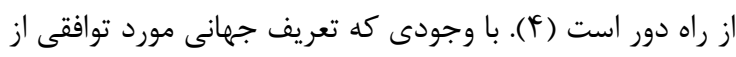

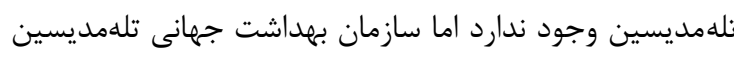

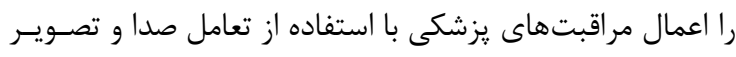

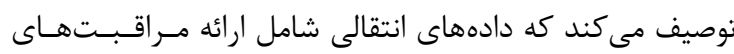

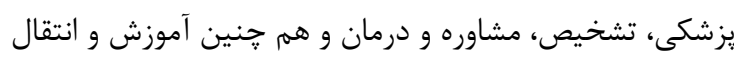

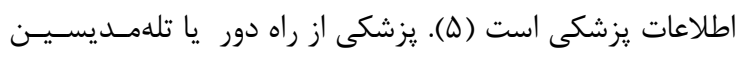

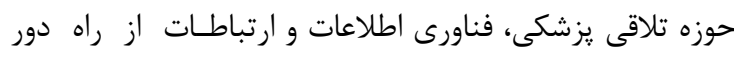

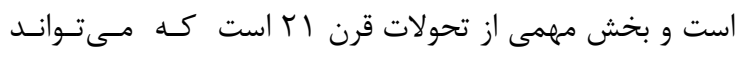
بزركترين تاثيرات رادر سيستم بهداشت و درمان داشته باشد (ه) (ه).

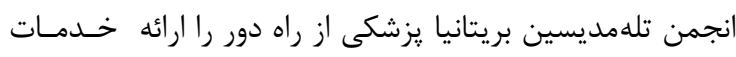

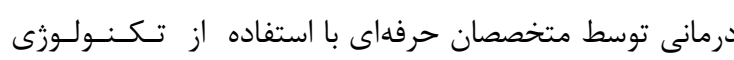

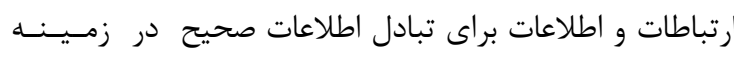

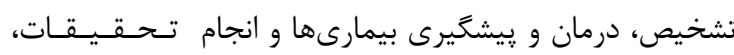

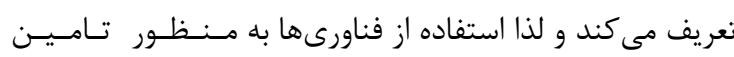

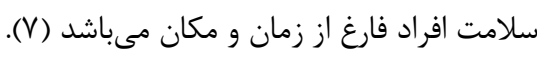

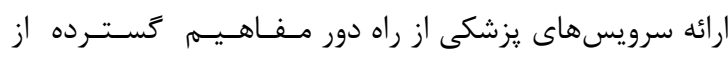

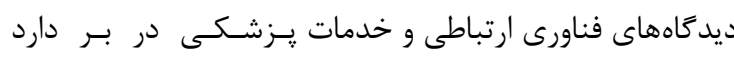

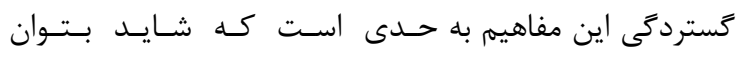

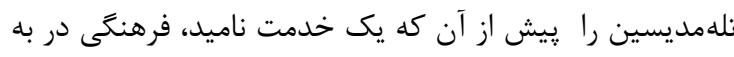

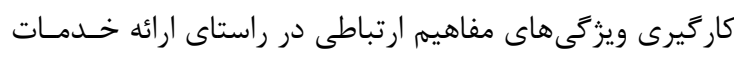

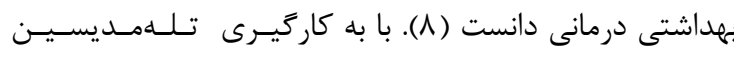

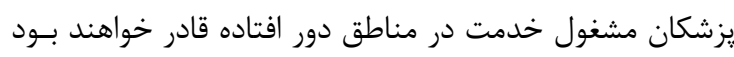

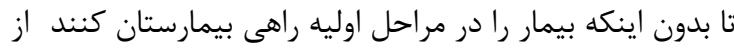

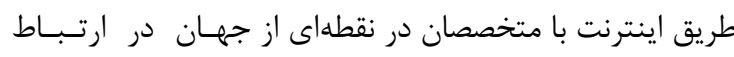

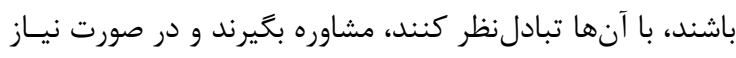

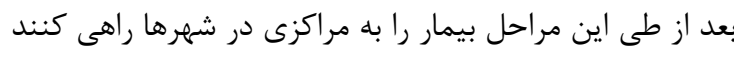

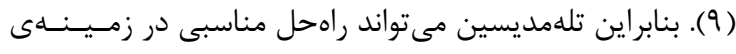
كمك هاى يزشكى فورى، مديريت و نظارت و تضمين كيفيت و

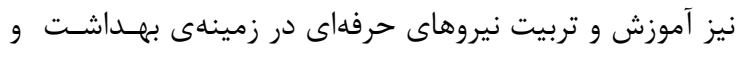

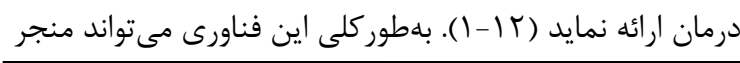

1- Telemedicine 


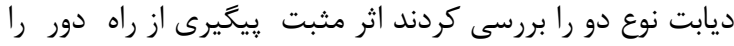

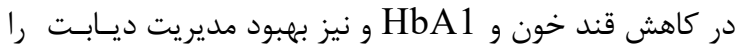

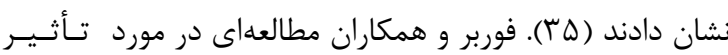

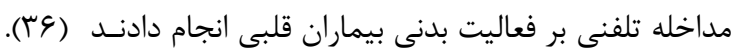

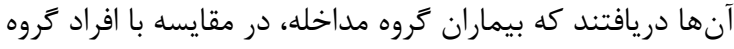

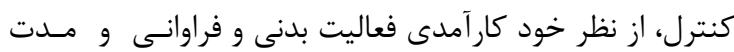

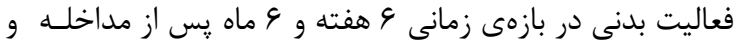

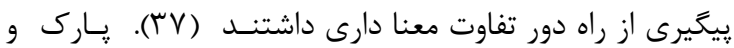

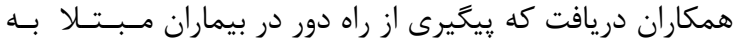

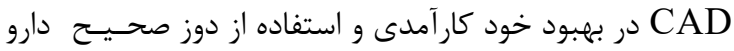

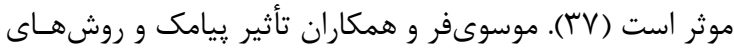

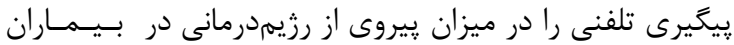

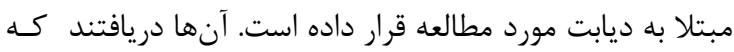

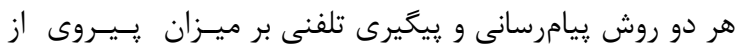

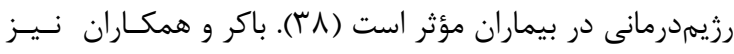

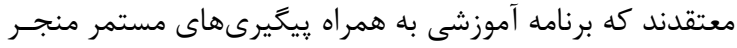

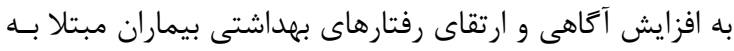

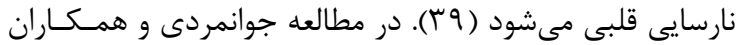

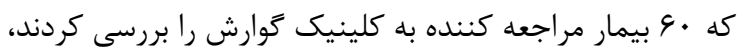

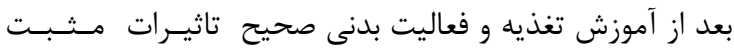

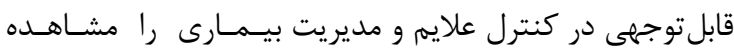

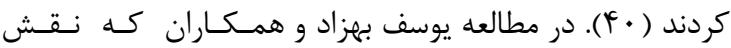

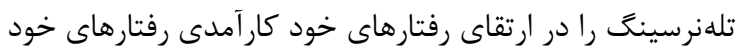

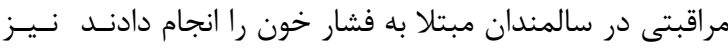

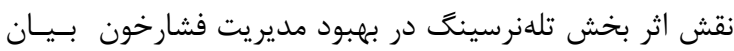

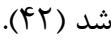

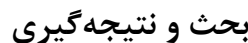

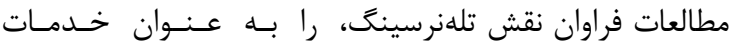

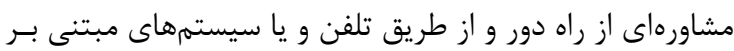

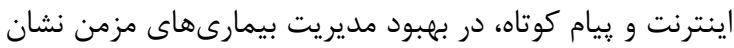

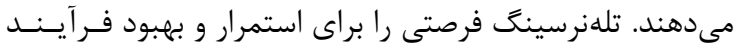

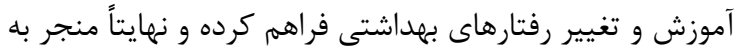

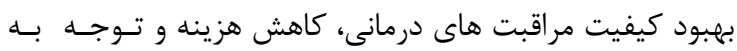

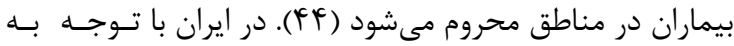

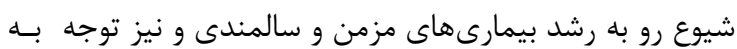

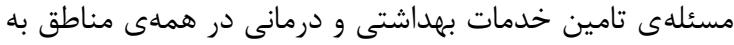

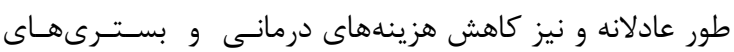

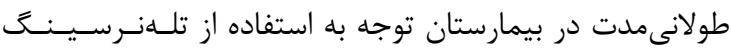

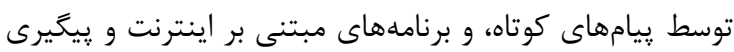

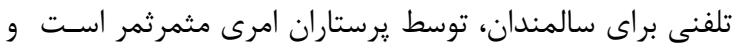

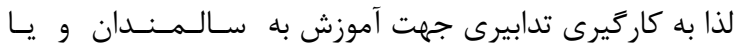

بنابر اين مطالعهى حاضر با هدف بررسى نقش تلفنرسيـــــ در

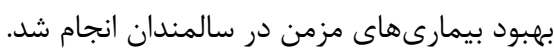

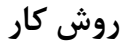

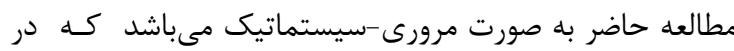

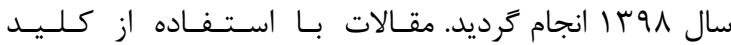

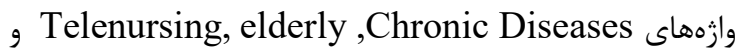
تركيب آنها، در پايخاههاى فارسى SID, Magiran و پإيـــاه

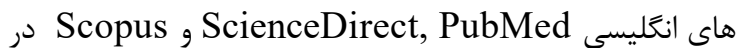

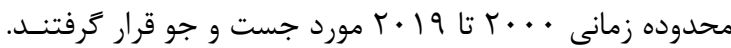

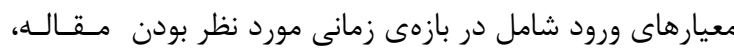

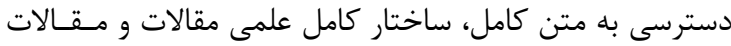

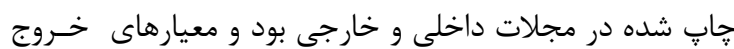

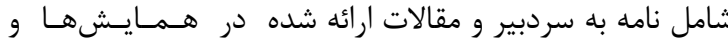

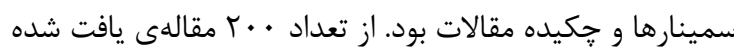

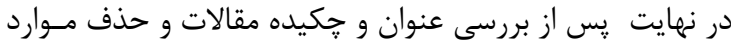

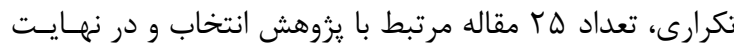

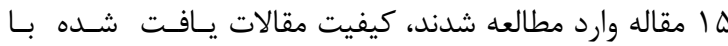

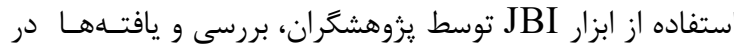
جإقى ليست مورد نظر وارد كرديد.

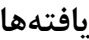

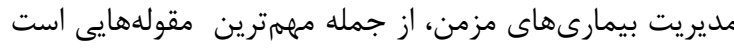

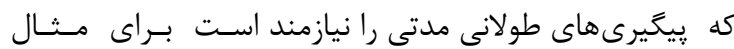

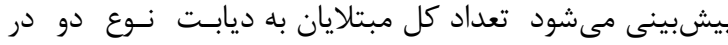

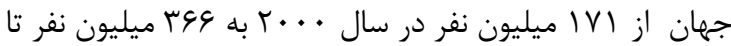

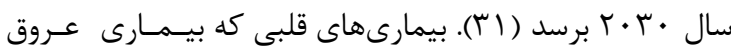

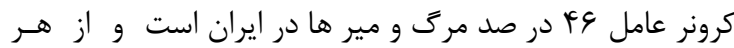

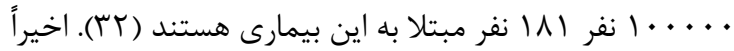

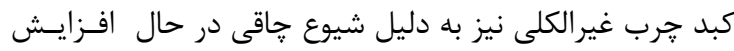

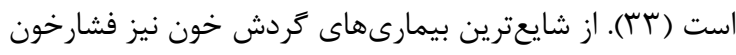

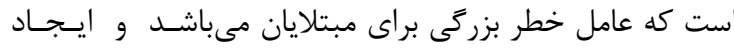

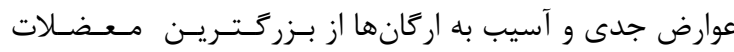

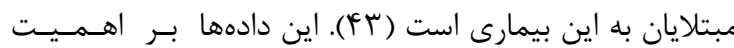

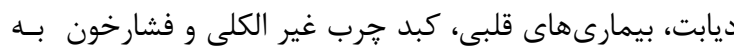
عنوان يك مشكل بهداشت جهانى، تاكيد مي كنند ( آسا). لذا در

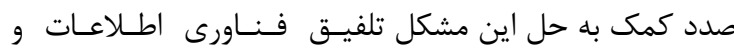

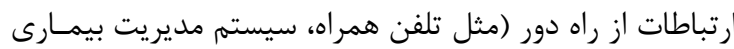

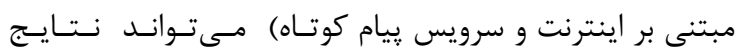

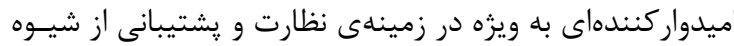

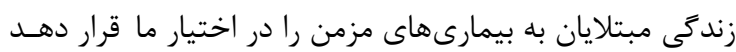

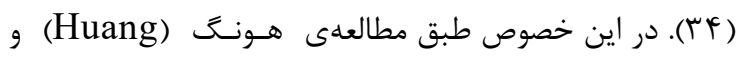

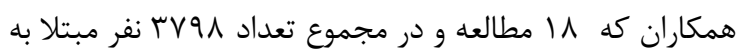

3- Furber 


\section{References}

1. Hyman WA. Telemedicine: Theory and Practice. In Bashshur RL, Sanders JH, Shannon GW, editors. Annals of Biomedical Engineering. 2000; 27 (2): 286

2. Khodadadeh $\mathrm{M}$, keshvari $\mathrm{H}$ et all, Review the requirements and legal challenges needed to tele medicine system 2016 Persian.

3. Wu ZY, Lee YC, Lai F, Lee HC, Chung Y. A secure authentication scheme for telecare medicine information systems.Journal of medical systems. 2012; 36(3):1529-35.

4. Zailani S, Gilani MS, Nikbin D, Iranmanesh M. Determinants of telemedicine acceptance in selected public hospitals in Malaysia: clinical perspective. J Med Syst. 2014; 38(9):111.

5. Adler A T. A Cost-effective PortableTelemedicine Kit for Use in DevelopingCountries, Massachusetts Institute of Technology,Cambridge, MA. 2000.

6. Taghipour M, AhmadzadehSh, KeshvariH, Imanzadeh M. [The use and implementation oftelemedical systems for the purpose of aerospacehealth monitoring]. Hospital 2013; 12(5):35-42. (Persian). 7. Sharifi MH. The use of telemedicine (telemedicine) in disaster. Third International Conference on crisis management in disasters. 2007, Tehran, promote quality companies, http:// www.civilica.com/Paper-INDM03-

INDM03 045.html. (Persian)

8. Oh H, Rizo C, Enkin M, Jadad A. What is eHealth?:A systematic review of published definitions. World Hosp Health Serv. 2005; 41(1):32-40.

9. Bafghizade $M$, Najarzadeh $Z$, Imanzadeh $M$. [Role of GIS in the establishment of optimal telemedicine system]. Hospital. 2013; 12(5):19-24. (Persian).

10. Stanberry B. Telemedicine: barriers and opportunities in the 21 st century. J Intern Med. 2000 Jun; 247(6):615-28.

11. Lin CC, Chen HS, Chen CY, Hou SM. Implementation and evaluation of a multifunctional telemedicine system in NTUH.Int J Med Inform. 2001 May; 61(2-3):175-87.

12. Hersh WR, Helfand M, Wallace J, et al. Clinical outcomes resulting from telemedicine interventions: a systematic review. BMC Med Inform DecisMak. 2001; 1:5.Epub 2001 Nov 26.

13. Nematolahi M, Abhari Sh. Assessing the Information and Communication Technology Infrastructures of Shiraz University of Medical Sciences in order to Implement the Telemedicine System in 2013. Journal of hospital 2014; 5(2): 44-51. (Persian)

14. Black JM, Hawks JH. Medical-surgical nursing: Saunders/Elsevier; 2009.

15. Schlachta-Fairchild L, Elfrink V, Deickman A. Patient Safety, Telenursing, and Telehealth. 2009.

16. Shoaei F, Nejati V. Elderly-caring service pattern in USA comparing with IRAN. Iranian Journal of Ageing. 2008; 3 (1):68-77.

17. HayaviHaghighi M, Alipour J, Mastaneh Z, Mouseli L. [Feasibility study of telemedicine implementation in Hormozgan university of medical sciences]. Bimonthly Journal of Hormozgan Uni-

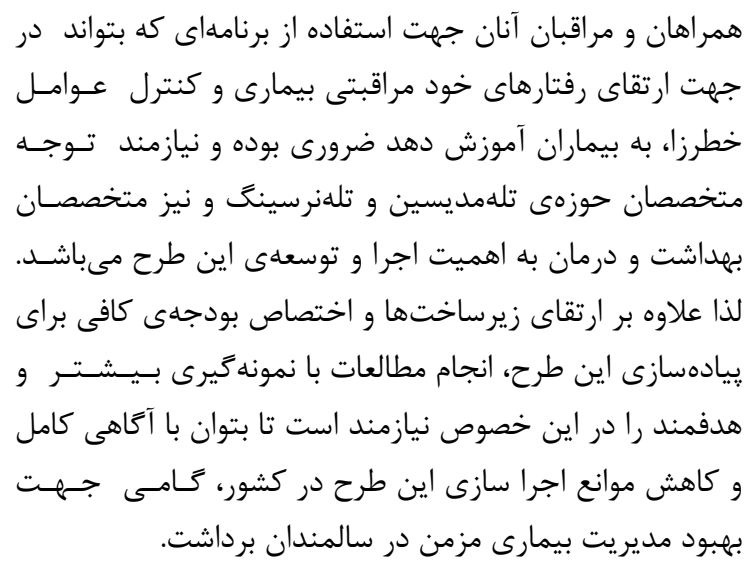


versity of Medical Sciences. 2011;15(2):128-37. Persian.

18. Edirippulige S, Smith AC, Young J, Wootton R. Knowledge, perceptions and expectations of nurses in e-health: results of a survey in a children's hospital. J Telemed Telecare. 2006;12(3_suppl):35 -8 .

19. Nagel DA, Pomerleau SG, Penner JL. Knowing, Caring, and Telehealth Technology: "Going the Distance" in Nursing Practice. J Holist Nurs. 2013; 31(2):104-12

20. Glinkowski W, Pawłowska K, Kozłowska L. Telehealth and telenursing perception and knowledge among university students of nursing in Poland. TELEMEDICINE and e-HEALTH. 2013;19(7):523-9.

21. Masjedi MR, Fadaizadeh L, Hosseini MS, EbrahimiKMR.Primary Experience of

Telemedicine at Shahid Beheshti University of Medical Sciences.Journal of Medical Council of Iran.2013; 31(2):113-8. Persian

22. Souza-Junior VD, Mendes IAC, Mazzo A, Godoy S. Application of telenursing in nursing practice: an integrative literature review. ApplNurs Res. 2016;29:254-60.

23. Tubaishat A, Al-Rawajfah OM, Habiballah L, Akhu-Zaheya LM. Exploring changes in

nursing students' attitudes towards the use of technology: A four-wave longitudinal panel study. Nurse Educ Today. 2016;38:101-6.

24. Darvish A, Bahramnezhad F, Keyhanian S, Navidhamidi M. [The role of nursing informatics On promoting quality of health care and the need for appropriate education]. Global journal of health science. 2014;6(6):11-18. (Persian)

25. Peck A. Changing the face of standard nursing practice through tele health and tele nursing .Nursing administration quarterly. 2005 Oct 1; 29 (4):339-43.

26. Alizadeh A, Mohammadi A, Khademloo M, Hosaini H. [Survey of Views of Medical

Students on Telemedicine Methods Developed in the Mazandaran University of Medical Sciences,

Iran]. Strides in Development of Medical Education.2013;10(2):247-59. Persian

27. Ahmadisahar et al, Comparing the effect of telephonic follow-up (tele-nursing) and face-toface on the sense of loneliness of covered elderly people Morocco Health of Ahwaz Urban Society Journal of Elderology 2013:2(2):58-65. (Persian)

28. Jennett P, Yeo M, Pauls M, Graham J. Organizational readiness for telemedicine: implications for success and failure. J Telemed Telecare 2003;9Suppl 2:S27-30.

29. Bahadorkhani M. Early study of employment telemedicine system in growth skill for university professors. 10th Congress of Medical Scinces; Ghazvin: Ghazvin University Of Medical Scincices; 2009. p. 5-7.

30- Gholamhoseini L, Sadeghi M, Mehrabi N. Aspects of telemedicine applications. Journal of Army university, Annals of military and health sciences research 2009; 3(1): 36-43. [Persian]

31. Wild S Roglic G Green A Sicree R King H. Global prevalence of diabetes: estimates for the year 2000 and projections for 2030 .

32. Boroumand S, Shahriari M, AbbasiJebeli M, Baghersad Z, Baradaranfard F, Ahmadpoori F. Determine the level of self-efficacy and its related factors in patients with ischemic heart disease: A descriptive correlational study. IJNR. 2015;9:61-9 33. Amali R, Jamali A. A Non-alcoholic fatty liver disease. Feyz, Journal of Kashan University of Medical Sciences. 2010;14:169-81. (Persian)

34. VanBerg N Schumann M Kraft K HoffmannW. Telemedicine and telecare for older patients - asystematic review. Maturitas20127394114

35. Huang Z, Tao H, Meng Q, Jiang L. Management of endocrine disease: Effects oftelecare intervention for glycemic control in Type 2 Diabetes: A systematicreview and meta-analysis of randomized controlled trials. Eur J Endocrinol2014; 172:R93R101.

36. Furber S, Butler L, Phongsavan P, Mark A, Bauman A. Randomised controlled trial of a pedometer-based telephone intervention to increase physical activity among cardiac patients not attending cardiac rehabilitation. Patient EducCouns. 2010;80:212-8

37. Park LG, Howie-Esquivel J, Chung ML, Dracup K. A text messaging intervention to promote medication adherence for patients with coronary heart disease: A randomized controlled trial. Patient EducCouns. 2014;94:261-8

38. Mousavifar SA, Zolfaghari M, Pedram SH, Haghani H. Examine the effectiveness of two methods follow-up (mobile and phone) on adherence to treatment in patients with diabetes. Iran J Diabetes Lipid Disord. 2011;10:407-18

39. Chang A. Six minute walking test. Australian Journal of Physiotherapy.2006; 52: 228-229.

40. JavanmardiFard S, Ghodsbin F, Kaviani MJ, Jahanbin I, Bagheri Z. The Effect of Follow up (Telenursing) on Liver Enzymes in Patients with Nonalcoholic Fatty Liver Disease: A Randomized Controlled Clinical Trial. Int J Community Based Nurs Midwifery. 2016; 4(3):239-46.

41. Javanmardifard S, Ghodsbin F, Kaviani MJ, Jahanbin I. The effect of telenursing on selfefficacy in patientswithnon-alcoholic fatty liver disease: a randomized controlled clinical trial. Gastroenterol.Hepatol Bed. Bench. 2017;10(4):263-71 42. Behzad Y, Bastani F, Haghani H. EFFECT OF EMPOWERMENT PROGRAM WITH THE TELEPHONE FOLLOW-UP (TELE-NURSING) ON SELF - EFFICACY IN SELF-CARE BEHAVIORS IN HYPERTENSIVE OLDER ADULTS. Nurs Midwifery J. 2016; 13 (11) :1004-1015. (Persian)

43. Rajaei S, Esmaeilzadeh A. The relationshipbetween the consumption of beverages withhypertension and serum uric acid level: A reviewof epidemiological evidence. Iran J Diabetes LipidDisorders 2010; 10(1):13-27. (Persian)

44. Askarikermani A, Khodayi M, Ansari A, Bahaedinbeygi $\mathrm{K}$. Propose a practical and simple way to consult Teleradiology, Journal of Kerman University of Medical Sciences 2012; 20(1): 102-108. (Persian) 